\title{
Cortical and trabecular bone are equally affected in rats with renal failure and secondary hyperparathyroidism
}

\author{
Nikita M. Bajwa ${ }^{1 \dagger}$, Cheryl P. Sanchez ${ }^{2 \dagger}$, Richard C. Lindsey ${ }^{1}$, Heather Watt ${ }^{1}$ and Subburaman Mohan ${ }^{1,3,4^{*}}$
}

\begin{abstract}
Background: Changes in mineral metabolism and bone structure develop early in the course of chronic kidney disease and at end-stage are associated with increased risk of fragility fractures. The disruption of phosphorus homeostasis leads to secondary hyperparathyroidism, a common complication of chronic kidney disease. However, the molecular pathways by which high phosphorus influences bone metabolism in the early stages of the disease are not completely understood. We investigated the effects of a high phosphorus diet on bone and mineral metabolism using a 5/6 nephrectomy model of chronic kidney disease.
\end{abstract}

Methods: Four-week old rats were randomly assigned into groups: 1) Control with standard diet, 2) Nephrectomy with standard rodent diet, and 3) Nephrectomy with high phosphorus diet. Rats underwent in vivo imaging at baseline, day 14 , and day 28 , followed by ex vivo imaging.

Results: Cortical bone density at the femoral mid-diaphysis was reduced in nephrectomy-control and nephrectomy-high phosphorus compared to control rats. In contrast, trabecular bone mass was reduced at both the lumbar vertebrae and the femoral secondary spongiosa in nephrectomy-high phosphorus but not in nephrectomy-control. Reduced trabecular bone volume adjusted for tissue volume was caused by changes in trabecular number and separation at day 35. Histomorphometry revealed increased bone resorption in tibial secondary spongiosa in nephrectomy-control. High phosphorus diet-induced changes in bone microstructure were accompanied by increased serum parathyroid hormone and fibroblast growth factor 23 levels.

Conclusion: Our study demonstrates that changes in mineral metabolism and hormonal dysfunction contribute to trabecular and cortical bone changes in this model of early chronic kidney disease.

Keywords: Chronic kidney disease, Bone, Renal pathology, Phosphorus, FGF23

\section{Background}

The incidence and prevalence of chronic kidney disease (CKD) continues to rise, with rates doubling in people aged 65 years or older [1]. The pathological manifestations of the disease include renal hyperparathyroidism, cardiovascular disease, vascular calcification, and renal osteodystrophy, all of which can alter bone mineral metabolism resulting in increased fracture risk and other skeletal abnormalities [2-5]. Patients with moderate CKD can have up to a 2.4-fold

\footnotetext{
* Correspondence: subburaman.mohan@va.gov

${ }^{\dagger}$ Equal contributors

${ }^{1}$ Musculoskeletal Disease Center, VA Loma Linda Healthcare System, 11201 Benton Street, Loma Linda, CA 92357, USA

${ }^{3}$ Department of Medicine, Loma Linda University, Loma Linda, CA 92354, USA Full list of author information is available at the end of the article
}

increase in fracture occurrence compared to mild CKD or healthy patients [6].

CKD causes a dysregulation of mineral homeostasis that negatively alters bone mineralization parameters and leads to skeletal abnormalities [7, 8]. Several rodent models have been developed for CKD to evaluate the consequence and underlying mechanisms for bone metabolism changes in response to moderate CKD. In a 5/6-nephrectomy mouse model of CKD, the material properties of cortical bone within the tibial diaphysis were affected [9]. Similarly, a 5/6-nephrectomy rat model of CKD altered bone mineral and material properties and led to hyperparathyroidism [10]. In another model of CKD where rats underwent thyroparathyroidectomy and partial nephrectomy, cortical bone mineral properties in the 
femur were altered resulting in reduced mechanical strength [11]. In patients with CKD, common complications include hyperphosphatemia and secondary renal hyperparathyroidism (rHPT), in which disturbances in the homeostasis of calcium, phosphate, and vitamin D lead to elevated parathyroid hormone (PTH) levels and bone loss. Elevated PTH levels lead to reduced cortical [3] and trabecular bone mineral densities [12] as well as pronounced changes in trabecular microstructure [12-16].

In the absence of disease, calcium and phosphorus levels are maintained through a complex interaction between the kidneys, intestine, bones, and parathyroid glands. Calciumsensing receptors in the parathyroid gland detect changes in serum calcium and regulate the synthesis and secretion of PTH [17]. Increases in PTH levels, as in CKD, occur as a response to increased renal phosphorus excretion and the downregulation of calcium sensing receptors that lead to sustained activation of PTH, thus causing detrimental effects on bone [18]. The disruption of phosphate homeostasis in CKD induces secondary hyperparathyroidism. Furthermore, higher levels of fibroblast growth factor 23 (FGF23), a bone-derived hormone that regulates phosphorous and $1,25[\mathrm{OH}]_{2} \mathrm{D} 3$, may in fact precede changes in PTH levels [19]. Calcium, $1,25[\mathrm{OH}]_{2} \mathrm{D} 3$, and PTH stimulate FGF23 secretion via negative endocrine feedback loops [20, 21]. Thus, PTH and FGF23 have been implicated as key players in mediating the observed changes in skeletal metabolism during kidney failure.

The effects of chronic renal failure on bone metabolism have been studied with nephrectomy animal models, and the $5 / 6$ nephrectomy model is commonly used to induce severe secondary hyperparathyroidism. The aim of this study was to evaluate the effect of high phosphorus diet on the trabecular and cortical bone in rats with kidney failure and advanced secondary hyperparathyroidism using DXA scanning and micro-CT. We also aimed to determine the relationship between skeletal changes and serum levels of PTH, FGF23, and sclerostin.

\section{Methods}

\section{Animals}

Four-week old male Sprague-Dawley rats (Charles River, San Diego, CA) were used for this study. Animal housing and procedures were approved by the Institutional Animal Care and Use Committee of the Jerry L. Pettis Memorial Veterans Affairs Medical Center. Rats were anesthetized with isoflurane prior to surgical procedures. All procedures performed followed the EU Directive 2010/63/EU for animal experiments. At the completion of the study, rats were anesthetized with pentobarbital, followed by cardiac puncture until euthanasia.

After an acclimation period of 1 week, rats were randomly assigned into the following 3 groups: 1) Control group fed with standard rodent diet (Control), 2) Nephrectomy group fed with standard rodent diet (Nx-Control), and 3)
Nephrectomy group fed with high phosphorus diet (NxPhos). The standard rodent diet contained $0.68 \%$ phosphorus and $0.6 \%$ calcium. The high phosphorus diet used to induce advanced secondary hyperparathyroidism contained 1.2\% phosphorus and $0.9 \%$ calcium. In order to maintain equal caloric intake between groups, the control group was pair fed to nephrectomized animals. Rats were maintained on these diets until completion of the study. Details of the experimental protocol are described in the text (Fig. 1a).

\section{Nephrectomy}

Rats were deeply anesthetized and maintained at $1.5-2.5 \%$ isoflurane with oxygen. The 5/6 nephrectomy was performed as previously described [22]. Briefly, $2 / 3$ of the left kidney was removed via a left paramedian incision on the back. The adrenal gland was carefully freed from the upper pole of the renal capsule before the renal pedicle was ligated and the kidney partially removed. Five days later, the right kidney was removed via a right paramedian incision on the back. For the control group, kidneys were manipulated without ablation. Rats were sacrificed under anesthesia 35 days after the first surgery. Kidneys from each animal were snap frozen in liquid nitrogen and stored at $80{ }^{\circ} \mathrm{C}$ for protein extraction.

\section{Serum analysis}

Blood was collected from rats on days 19 and 26 postnephrectomy. Serum was stored at $-80^{\circ} \mathrm{C}$ for future quantitation of intact FGF23 (EMD Millipore, Temecula, CA), phosphorus (Biovision Inc., Milpitas, CA), sclerostin (R\&D Systems, Minneapolis, MN), calcium (Fisher Scientific, Hampton, $\mathrm{NH}$ ), creatinine (Biovision Inc., Milpitas, $\mathrm{CA}$ ), and intact PTH (iPTH) (Fisher Scientific, Hampton, NH) with ELISA kits. ELISAs were performed according to manufacturers' recommended instructions.

\section{Evaluation of bone phenotypes}

Cortical bone density and bone area of the total body, femur, tibia, and lumbar vertebrae (L4-6) were measured by dual-energy X-ray absorptiometry (DXA) using a PIXImus instrument (Lunar Corp) according to previously published methods [23, 24]. Trabecular and cortical bone parameters of the femur and L 5 vertebrae were analyzed by microcomputed tomography (micro-CT; VIVA CT40, SCANO Medical) according to previously published methods $[25,26]$. Micro-CT scanning was performed with $55-70 \mathrm{kVp}$ ( $55 \mathrm{kVp}$ for trabecular bone, $70 \mathrm{kVp}$ for cortical bone) and a voxel size of $10.5 \mu \mathrm{m}$, and microarchitecture reconstructions were performed using the SCANCO software (SCANCO Medical).

\section{Histomorphometry}

The tibiae were fixed and processed as previously reported [27]. Data were analyzed using the OsteoMeasure software 
a

\section{Timeline}

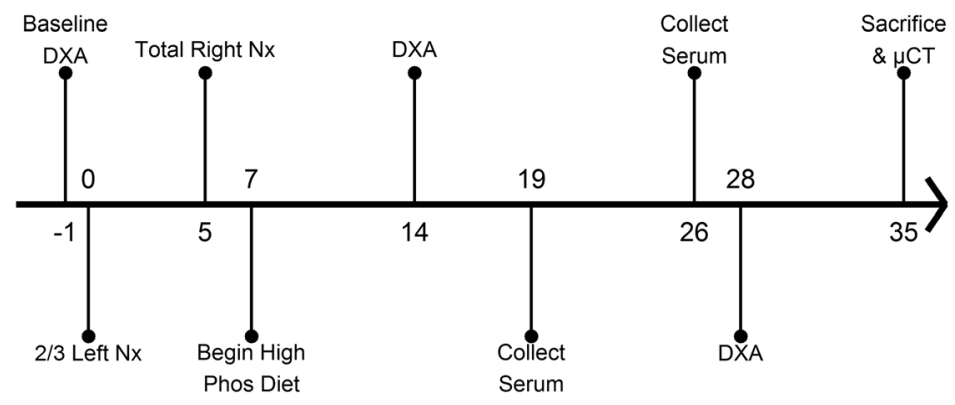

Day
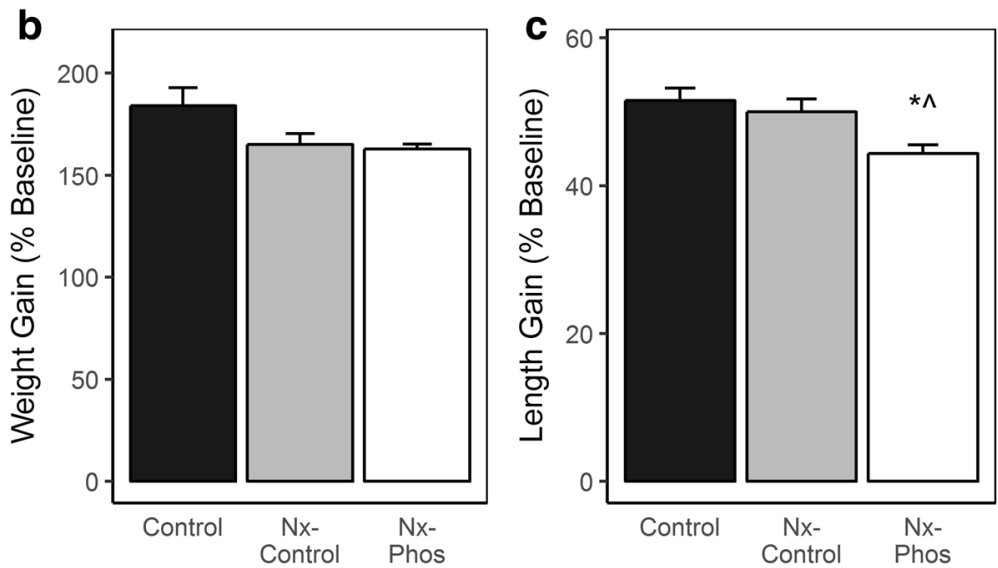

Fig. 1 Study timeline and the effect of phosphorus on bone measurements. (a) Rats underwent baseline in vivo DXA imaging, followed by partial nephrectomy of the left kidney at day 0 . At day 5 , rats underwent total nephrectomy of the right kidney and began a high phosphorus diet or standard diet at day 7. In vivo DXA imaging occurred at 14 days post-nephrectomy, followed by serum collection 5 days later. One week later, serum was collected again, followed by in vivo DXA imaging at 28 days post-nephrectomy. At 35 days, rats were euthanized and bones underwent ex vivo micro-CT imaging. (b) Weight was monitored weekly throughout the duration of the study. Values are presented as mean percent increase over baseline \pm SEM; $n=6-8$. (c) Body length was obtained at baseline, prior to surgery and euthanasia. Values are presented as mean percent increase over baseline \pm SEM; $n=6-8$. *Significant at $p<0.05$ versus Control animals. ^Significant at $p<0.05$ versus Nx-Control animals

(Osteometrics, Inc., Decatur, GA, USA), and measurements were taken at 10X magnification. Bone resorption in the secondary spongiosa of the distal tibia was measured with tartrate-resistant acid phosphatase (TRAP) staining. Oc.S/BS and Oc.Pm were measured according to established methods [28].

\section{Results}

All rats survived to the completion of the study. There was no significant difference in body weight
(Fig. 1b) between groups, but gain in total body length was reduced by $7.6 \%(p<0.05)$ in the Nx-Phos vs. control and by $6.5 \%(p<0.05)$ in the Nx-Phos vs. $\mathrm{Nx}$-Control (Fig. 1c). See Table 1 for anthropometric measurements.

As expected, both femur and lumbar bone mineral density (BMD) increased across time in all 3 groups of rats. However, the lumbar BMD was significantly lower in the $\mathrm{Nx}$-Phos compared to $\mathrm{Nx}$-Control and control groups at 2 and 4 weeks post-surgery $(p<0.01$; Fig. $2 \mathrm{a})$. After 2 weeks, femur BMD was significantly reduced in

Table 1 Anthropometric measurements

\begin{tabular}{llllll}
\hline Group & Initial Weight $(\mathrm{g})$ & Final Weight $(\mathrm{g})$ & Initial Body Length $(\mathrm{cm})$ & Final Body Length $(\mathrm{cm})$ & Final Femur Length $(\mathrm{cm})$ \\
\hline Control & $95.77 \pm 2.93$ & $267.5 \pm 4.0$ & $25.09 \pm 0.26$ & $38.07 \pm 0.03$ & $3.164 \pm 0.035$ \\
Neph-Control & $97.60 \pm 1.74$ & $260.9 \pm 4.7$ & $25.51 \pm 0.28$ & $38.35 \pm 0.09$ & $3.151 \pm 0.027$ \\
Neph-Phos & $99.34 \pm 1.30$ & $257.6 \pm 4.4$ & $26.11 \pm 0.23$ & $38.18 \pm 0.04$ & $3.100 \pm 0.025$ \\
\hline
\end{tabular}

Values are presented as means \pm SEM 


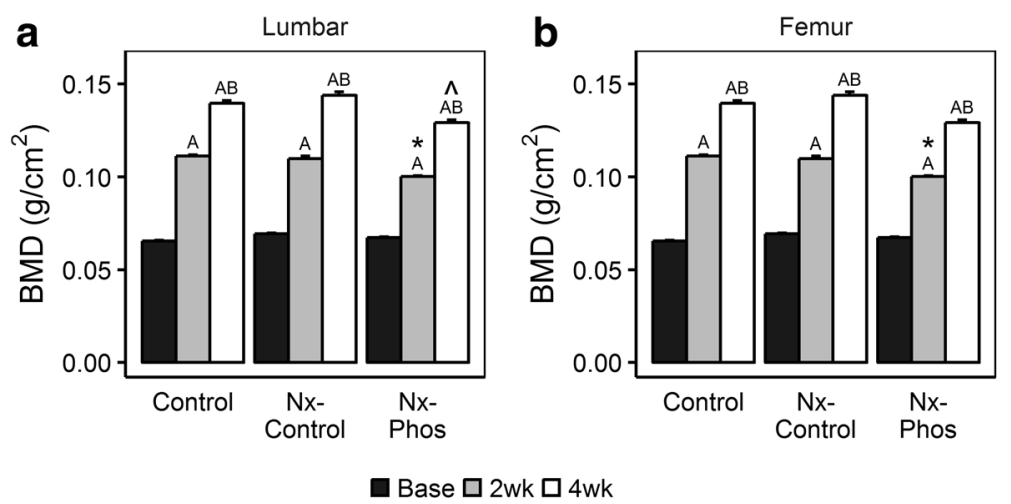

Fig. 2 Effects of nephrectomy and/or high phosphorus diet on lumbar and femoral bone mineral density (BMD). (a) BMD was determined from DXA analysis of the lumbar vertebrae. ${ }^{A}$ Significant at $p<0.01$ versus baseline rats. ${ }^{B}$ Significant at $p<0.01$ versus 2 weeks post-nephrectomy. ${ }^{*}$ Significant at $p<0.05$ versus Control. $\wedge$ Significant at $p<0.01$ versus Nx-Control. Values are presented as means $\pm \mathrm{SEM} ; n=11-14$. (b) BMD was determined from DXA analysis of the femur. ASignificant at $p<0.01$ versus baseline rats. ${ }^{B}$ Significant at $p<0.01$ versus 2 weeks post-nephrectomy. ${ }^{*}$ Significant at $p<0.05$ versus Control. Values are presented as means \pm SEM; $\mathrm{n}=11-14$

the Nx-Phos group compared to the other groups $(p<$ 0.05 ; Fig. 2b) but not at 4 weeks post-surgery

Micro-CT analysis showed that nephrectomy triggered significant cortical bone loss. The volumetric bone mineral density (vBMD) in the fifth lumbar vertebrae was reduced by $4.5 \%$ in $\mathrm{Nx}$-Control $(p<0.01)$ and $4.2 \%$ in $\mathrm{Nx}$-Phos $(p<0.01)$ vs. Control (Fig. 3a). Femur cortical vBMD was also reduced by $2.6 \%$ in Nx-Control $(p<0.01)$ and $3 \%$ in Nx-Phos $(p<.01)$ vs. Control group (Fig. $3 \mathrm{~b})$. Micro-CT analyses of trabecular bone of L5 vertebrae revealed that trabecular bone volume adjusted for tissue volume (BV/TV) was significantly reduced in $\mathrm{Nx}-\mathrm{Phos}$ compared to $\mathrm{Nx}$-Control and Control animals $(p<0.01$; Fig. $4 \mathrm{a}-\mathrm{c})$. The reduced trabecular BV was caused by significant reductions in trabecular number (Fig. 4d) and increases in trabecular separation (Fig. 4e). In contrast, trabecular thickness was not different among the three groups of rats (Fig. 4f). The loss of trabecular elements reduced the number of interconnections between trabeculae as reflected by a $>25 \%$ reduction $(p<0.01)$ in connectivity density in the Nx-Phos compared to Nx-Control and Control groups (Fig. 4g). Accordingly, structure model index (SMI), which indicates relative prevalence of rod-like to plate-like structures, was increased in Nx-Phos animals (Fig. 4h). Similar to lumbar vertebrae, femurs of Nx-Phos animals exhibited significantly reduced trabecular BV that was caused by changes in trabecular number and thickness (Fig. 5a-g). Consistent with the changes in trabecular architecture, connectivity density was decreased while SMI was increased in the femurs of Nx-Phos animals (Fig. 5h-i). In contrast to bone volume (BV), measured tissue volume (TV) was not different between the three groups at the lumbar vertebrae and femurs (Fig. 4b and Fig. 5c).

Histomorphometric measurements at the secondary spongiosa of the tibia revealed increased bone resorption (TRAP-positive surface) caused by nephrectomy (Table 2). TRAP-positive osteoclast surface adjusted for bone surface (Oc.S/BS) was increased by $177 \%(p<.01)$
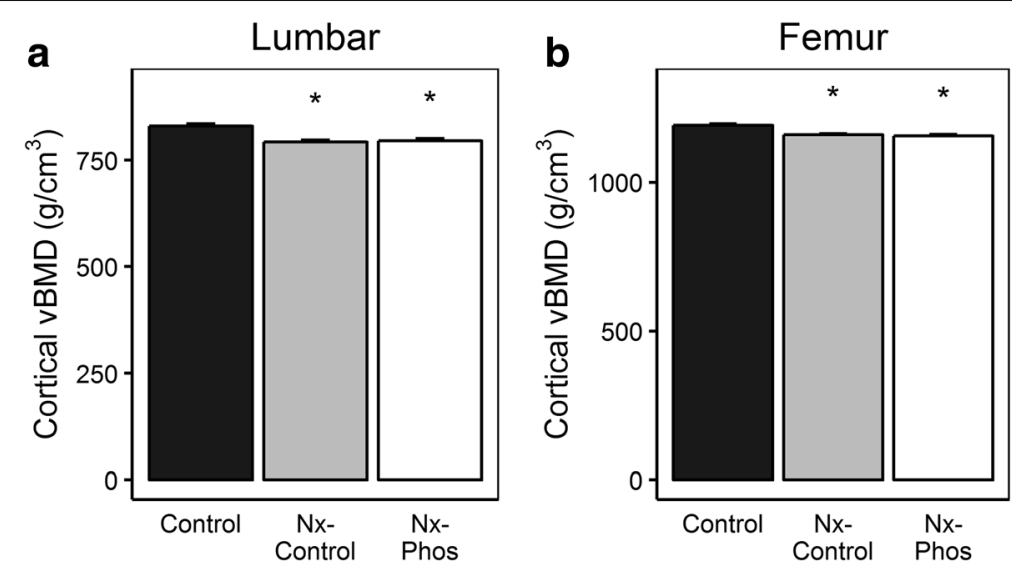

Fig. 3 Effects of nephrectomy and/or high phosphorus diet on cortical bone. The (a) lumbar vertebrae and (b) femur vBMD were determined from micro-CT analysis. *Significant at $p<0.01$ versus control. Values are presented as means \pm SEM; $n=10-12$ 


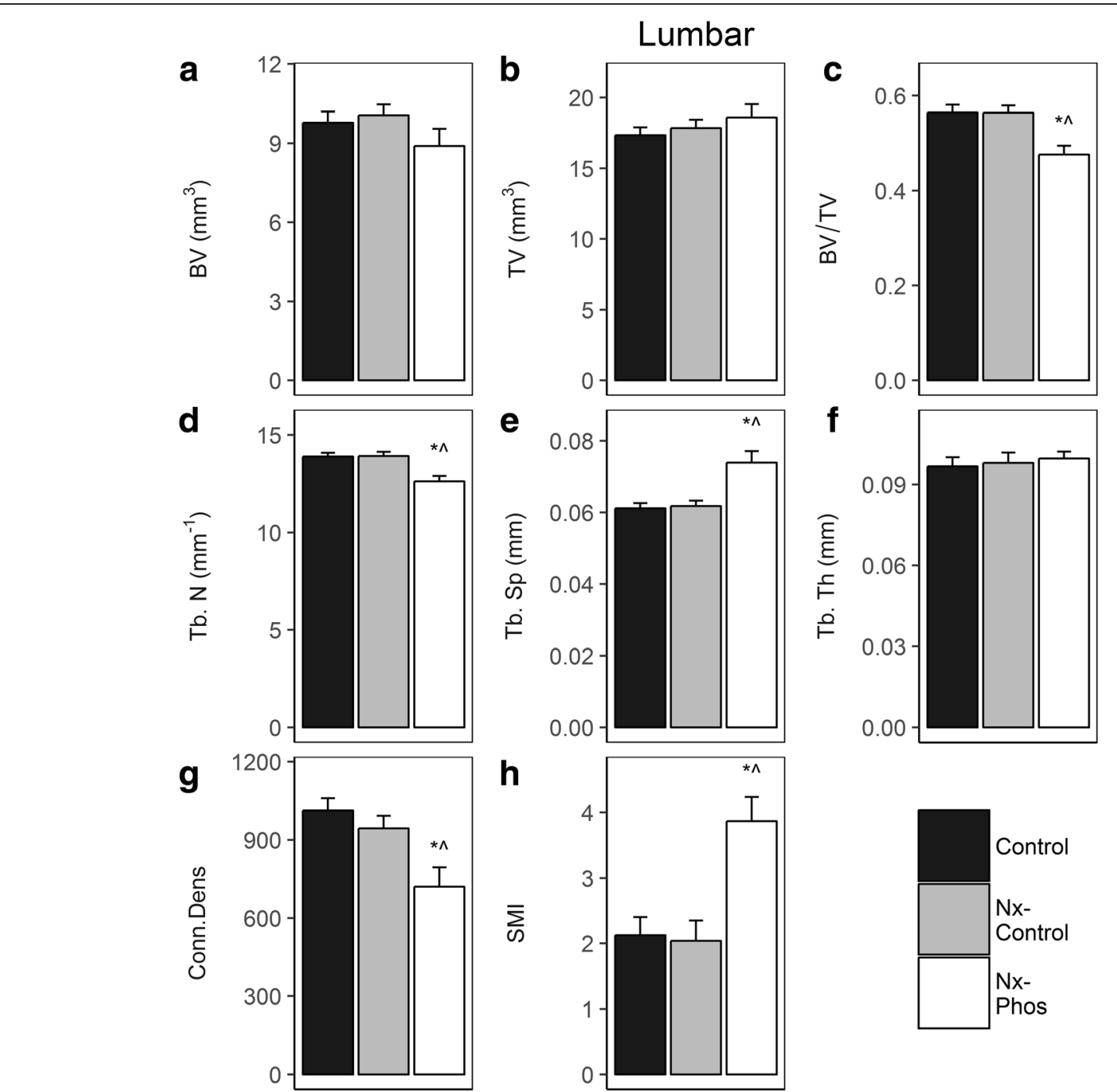

Fig. 4 Effects of nephrectomy and/or high phosphorus diet on the microstructure of lumbar bone. (a) Bone volume, (b) total volume, (c) total volumetric BMD (BV/TV), (d) trabecular number, (e) trabecular separation, (f) trabecular thickness, (g) connectivity density, and (h) structure model index were determined from micro-CT analysis. *Significant at $p<0.01$ versus Control. ^Significant at $p<0.01$ versus Nx-Control. Values are presented as means \pm SEM; $n=10-12$

in $\mathrm{Nx}-\mathrm{C}$ rats compared to Control. The Oc.S/BS was also increased by $72 \%(p<.05)$ in $\mathrm{Nx}$-Control compared to Nx-Phos. Similarly, the osteoclast perimeter (Oc.Pm) was also increased in $\mathrm{Nx}$-Control compared to Control rats, but did not reach statistical significance $(p=.08)$.

Table 2 Histomorphometric measurements at the secondary spongiosa of the tibia

\begin{tabular}{lll}
\hline Group & Oc.Pm & Oc.S/BS \\
\hline Control & $0.63 \pm 0.18$ & $5.76 \pm 1.63$ \\
Neph-Control & $1.12 \pm 0.19^{\#}$ & $15.95 \pm 2.86^{*}$ \\
Neph-Phos & $0.72 \pm 0.21$ & $9.25 \pm 1.46 \wedge^{@}$ \\
\hline
\end{tabular}

Values are presented as means \pm SEM. Oc.Pm represents osteoclast perimeter; Oc.S/BS represents osteoclast surface adjusted for bone surface; ${ }^{\#} p=.08$ versus control; * $p=0.006$ vs. control; $\wedge p=0.13$ versus control; ${ }^{@} p=0.04$ versus Neph-Control
Serum levels of calcium (Fig. 6a) and sclerostin (Fig. 6b) were unaffected in all groups at days 19 and 26 after nephrectomy. Serum phosphorus $(p<0.01$; Fig. $6 c)$, creatinine $(p=.05$; Fig. $6 \mathrm{~d})$, and FGF23 ( $p<0.01$; Fig. $6 \mathrm{e})$ levels were significantly elevated in $\mathrm{Nx}$-Phos animals at day 26 postsurgery. In addition, nephrectomy induced a significant increase in serum creatinine and iPTH at day 19 postsurgery. The increases in serum PTH levels at day 26 were significant for the $\mathrm{Nx}$-Phos but not for $\mathrm{Nx}$-Control animals $(p<0.01$; Fig. 6f). Serum levels of iPTH and FGF23 were negatively associated with trabecular vBMD $\left(r=-0.78, r^{2}=0.61, p<0.05 ; r=-0.78, r^{2}=0.61, p<.05\right.$; Fig. 7a \& b, respectively) at day 26 after nephrectomy. Serum levels of phosphorus trended towards a positive association with iPTH $\left(r=0.54, r^{2}=0.29, p=0.10\right.$; Fig. $\left.7 \mathrm{c}\right)$ at day 26 after nephrectomy. A positive relationship between 
a

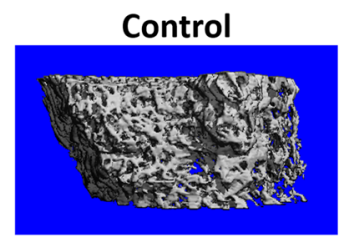

b

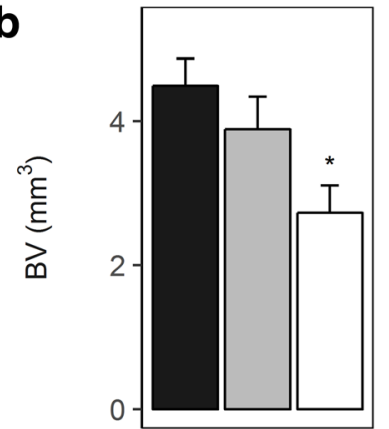

e

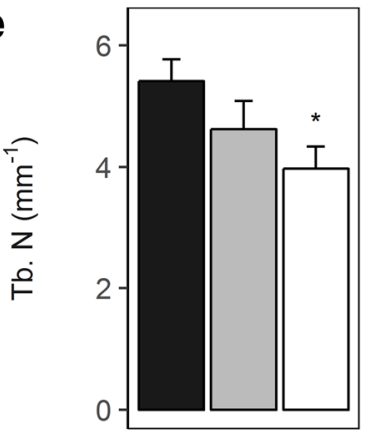

h

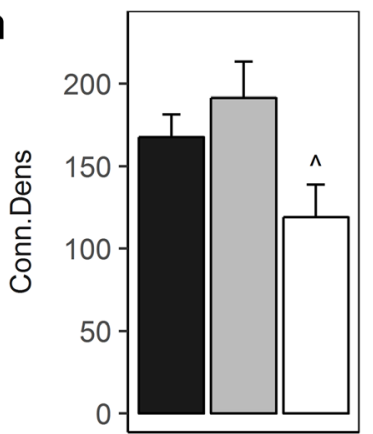

Nx-Control

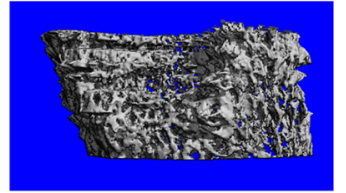

Femoral Trabecular
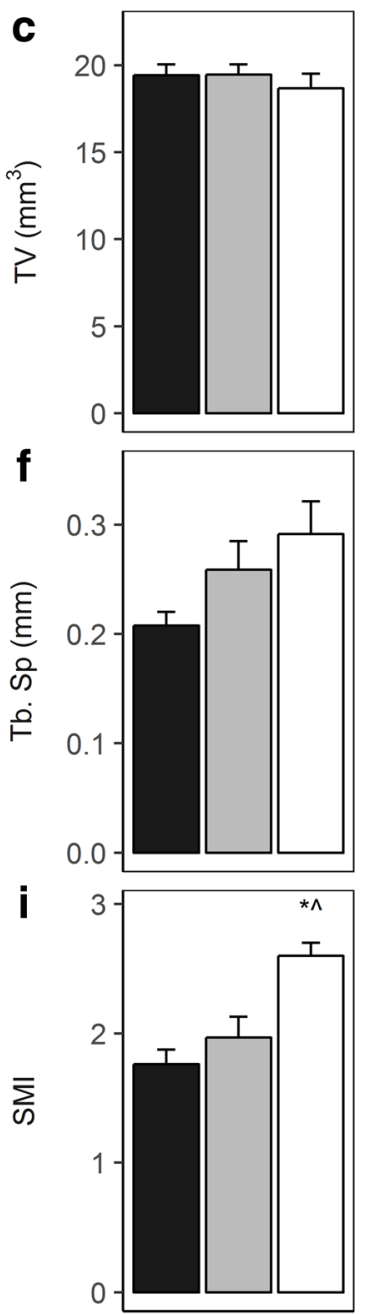

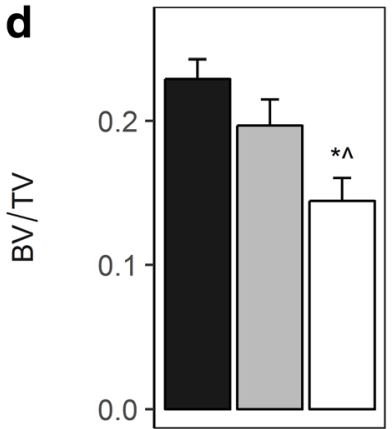

Nx-Phos

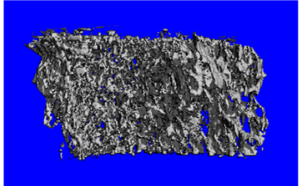

g

0.100
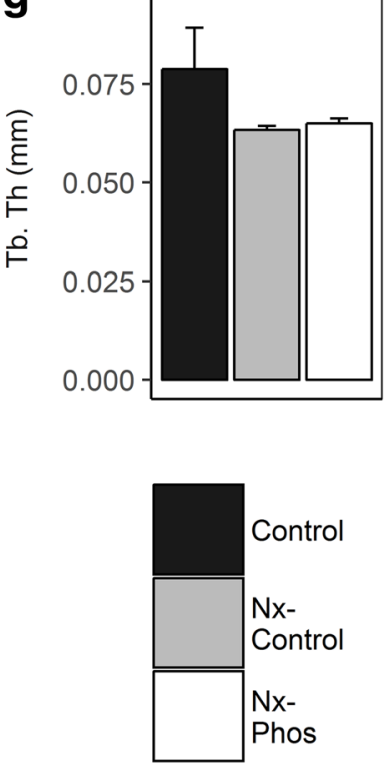

Fig. 5 Trabecular bone parameters of the femur. (a) Representative micro-CT images, (b) bone volume, (c) total volume, (d) total volumetric BMD (BV/TV), (e) trabecular number, (f) trabecular separation, (g) trabecular thickness, (h) connectivity density, and (i) structure model index were obtained from microCT analysis. *Significant at $p<0.01$ versus Control. $\wedge$ Significant at $p<0.01$ versus Nx-Control. Values are presented as means \pm SEM; $\mathrm{n}=10-12$

iPTH and FGF23 $\left(r=0.75, r^{2}=0.56, p<0.05\right.$; Fig. 7d $)$ was also observed at day 26 after nephrectomy. Interestingly, serum levels of sclerostin did not show significant correlation with bone parameters (trabecular or cortical bone), PTH, or FGF23 (data not shown).

\section{Discussion}

Animal models of CKD use nephrectomy (partial or complete) as a method to induce early renal failure to accelerate endocrine dysfunction and bone turnover.
Previous studies have shown the cortical region of bone to be the most susceptible to resorption in CKD [29, 30]. In this study, we investigated the effects of a high phosphorus diet on bone in a rat model of CKD and found that high phosphorus diet with advanced secondary hyperparathyroidism enhanced trabecular bone loss in the lumbar vertebrae and femur.

High levels of phosphorus have been found to increase vascular calcification, reduce cortical bone density and thickness, and cause severe trabecular bone alterations 

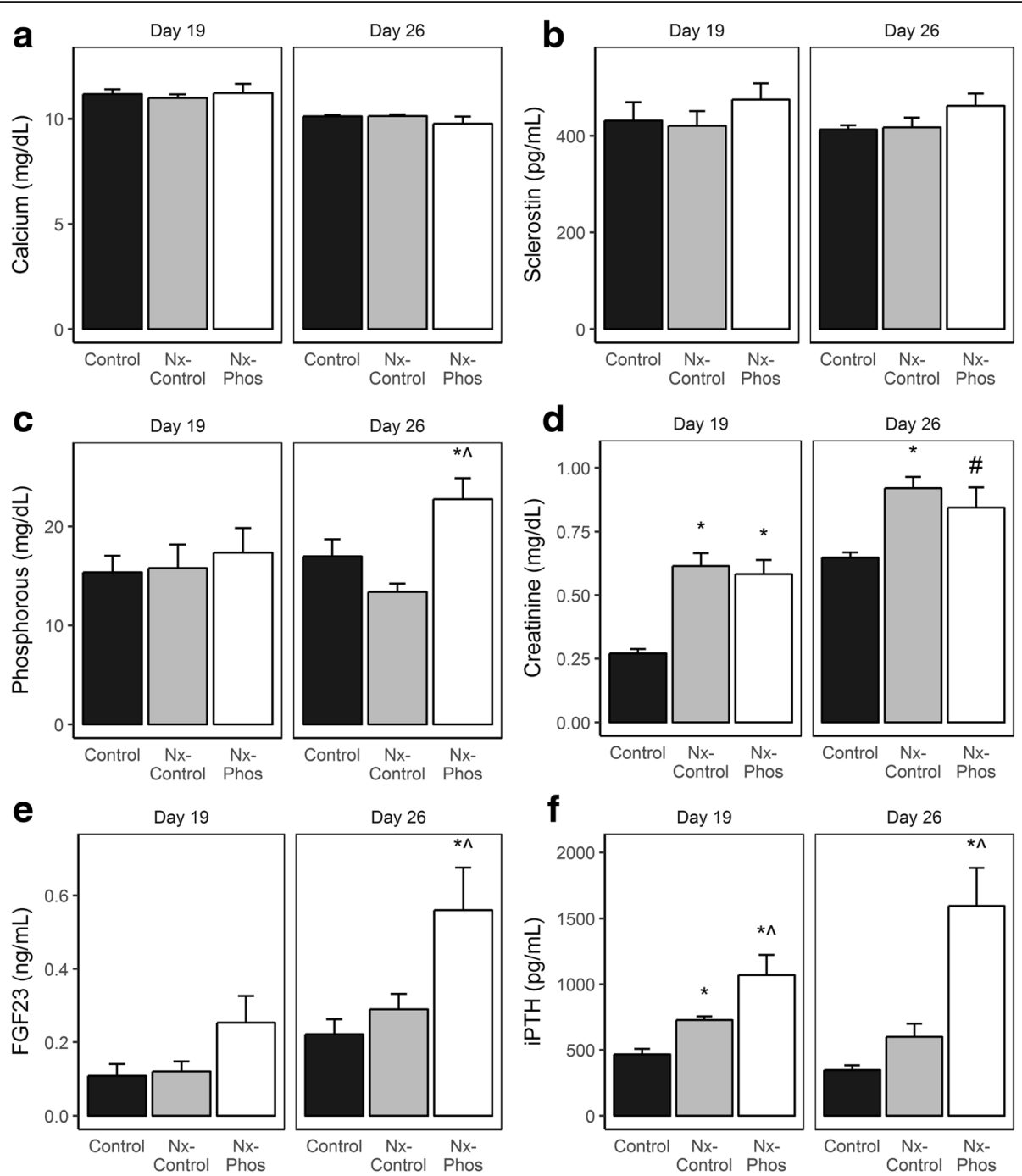

Fig. 6 Effects of nephrectomy and/or high phosphorus diet on serum levels of systemic bone formation and skeletal markers. Serum levels of (a) Calcium, (b) sclerostin, (c) phosphorus, (d) creatinine, (e) FGF23, and (f) iPTH were measured at days 19 and 26 post-nephrectomy. *Significant at $p<0.01$ versus Nx-Control. $\wedge$ Significant at $p<0.01$ versus Nx-Control. "Significant at $p<0.06$ versus Nx-Control. Values are presented as means \pm SEM; $n=3-9$

[2]. In a rat model of 5/6 nephrectomy, high phosphate reduced trabecular bone volume in the femur [31]. Similarly, we found that high phosphorus diet led to a significant change in bone microarchitecture as indicated by reduced trabecular bone volume, reduced trabecular number, and increased trabecular separation at day 35 . These changes were also present in the lumbar vertebrae where the reductions in trabecular bone volume were caused primarily by increased trabecular separation. The changes in trabecular bone parameters cannot be explained based on systemic changes in somatic growth as neither body weight nor femur length were significantly different among the three groups.

To further explore the mechanisms behind trabecular bone loss, we examined the relationship between serum levels of PTH and skeletal alterations induced by high phosphorus diet. We found that high phosphorus diet increased PTH levels at days 19 and 26 and that increased PTH could have contributed to the increased bone resorption and reduced trabecular bone mass. PTH is known to enhance both bone formation and resorption [32]. In addition to exerting anabolic effects on osteoblasts, PTH treatment increases expression of receptor activator of nuclear factor kappa-B ligand, a key regulator of osteoclastic differentiation and activation [32]. PTH also promotes the conversion of 25-hydroxy vitamin D3, an inactive form of vitamin $\mathrm{D} 3$, into active 1,25-dihydroxy vitamin D3 $\left(1,25[\mathrm{OH}]_{2} \mathrm{D} 3\right)$ by the activation of $1-\alpha-$ hydroxylase in kidneys [33] leading to absorption of calcium and phosphorous in the gut. Several studies have investigated how PTH and $1,25[\mathrm{OH}]_{2} \mathrm{D}$ affect 

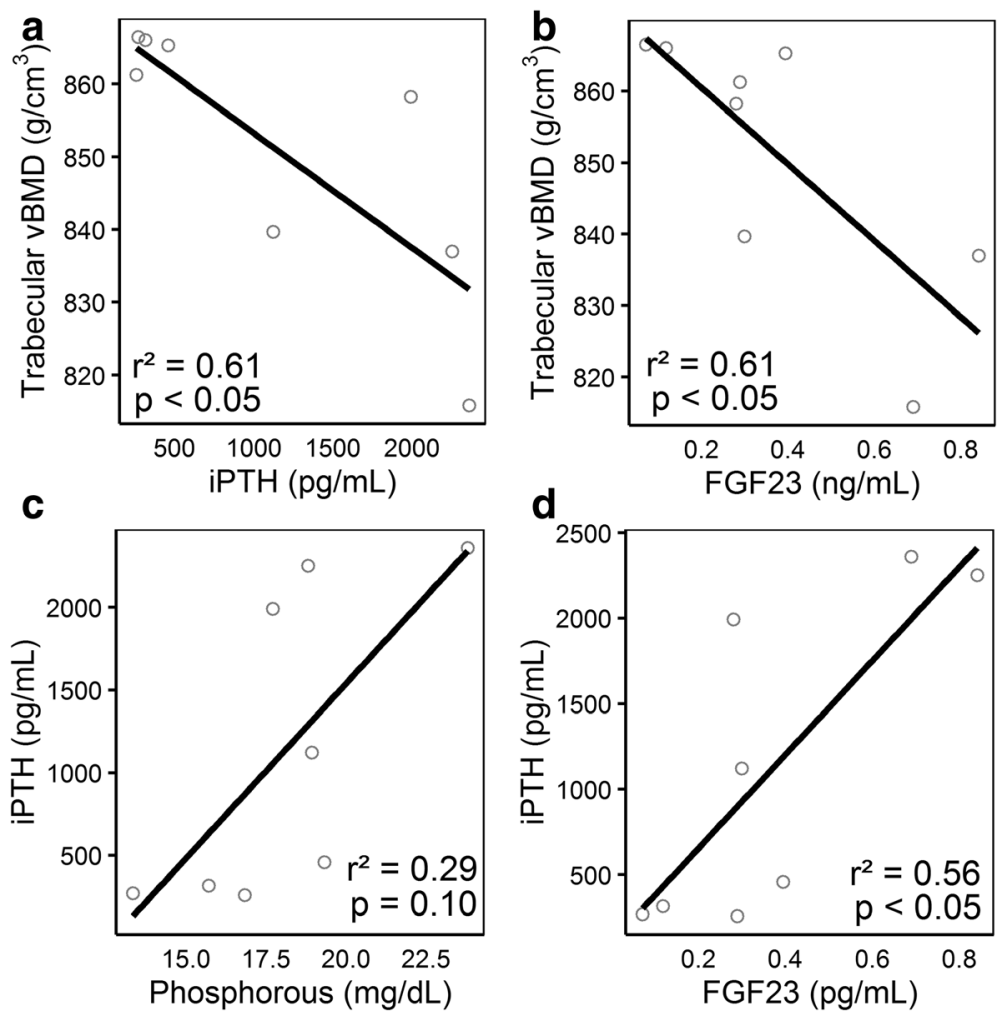

Fig. 7 Relationships between bone and serum levels of systemic bone formation and skeletal markers in rats with nephrectomy and/or high phosphorus diet. (a) Trabecular volumetric bone mineral density (vBMD) vs. iPTH, $\left(r^{2}=0.61, p<0.05\right)$, (b) trabecular vBMD vs. FGF23, $\left(r^{2}=0.61, p<0.05\right)$, (c) phosphorus vs. iPTH, $\left(r^{2}=0.29, p=.10\right)$, and (d) iPTH vs. FGF23, $\left(r^{2}=0.56, p<0.05\right)$ at day $26 ; n=8$

serum phosphate via calcium regulation [34, 35]. Our data shows that nephrectomy alone increased serum PTH levels. However, this increase in PTH was further augmented by high phosphorus diet and confirmed by a positive correlative relationship. We also do report that serum levels of iPTH and FGF23 were negatively associated with trabecular bone volume; however, the issue of whether elevated PTH contributes to both cortical bone loss caused by nephrectomy and trabecular bone loss caused by high phosphorus diet in the nephrectomy rats remains to be evaluated. There are also data to suggest that there may be alternate mechanisms by which high phosphate affects trabecular bone, regardless of PTH levels [31].

To explore alternate mechanisms, we also examined the relationship between serum levels of FGF23 and skeletal parameters in the combined data from the 3 groups of animals. FGF23 has been reported to play a role in balancing mineral ion homeostasis and bone mineralization through the regulation of phosphorus [36, 37]. FGF23 is known to be secreted by osteocytes and regulate phosphorus homeostasis by reducing the reactivity of sodiumphosphate co-transporters in the proximal renal tubules and affecting $1,25[\mathrm{OH}]_{2} \mathrm{D}$ metabolism and absorption by decreasing 1- $\alpha$-hydroxyase activity [37-40], thus excreting excess phosphate through the kidney. In CKD and renal failure, FGF23 concentrations rise to excrete excess phosphate as a result of diminished renal function, leading to high bone turnover. We found that high phosphorus diet increased serum FGF23 levels at day 26. Additionally, high levels of serum FGF23 were strongly associated with trabecular bone loss. In our study, elevations in FGF23 were associated with increased phosphate and PTH levels, and all parameters were negatively associated with bone density, as other studies have reported [41, 42]. Sclerostin, like FGF23, is mainly secreted by osteocytes [43, 44]. Sclerostin decreases bone formation by inhibiting terminal differentiation of osteoblasts and promoting their apoptosis [45] in part via blockade of Wnt signaling pathways by binding to LRP5/6 receptors $[45,46]$. In our studies, we did not find significant changes in serum levels of sclerostin in nephrectomy rats fed with normal or high phosphorus diet compared to control animals. However, we cannot exclude the possibility that local expression of sclerostin in osteocytes is altered by nephrectomy and/or high phosphorus diet. A recent study has also identified that FGF23/Klotho complex induces Dkk1 expression and thereby inactivates $\mathrm{Wnt} / \beta$-catenin signaling in osteoblasts. Thus, impairment of $\mathrm{Wnt} / \beta$-catenin signaling by 
high FGF23 levels may provide a mechanism for inhibition of bone formation in CKD [47]. In addition, the creatinine levels observed in our study are consistent with what others have reported in similar rat models of CKD [48-50].

Interestingly, nephrectomy induced significant cortical bone loss and higher osteoclast activity in the secondary spongiosa of the tibia, independent of phosphorus intake. Impaired kidney function may have altered the mineral or collagen composition of the cortical bone matrix [11, 51], which, in combination with PTH levels, may increase porosity [7]. In fact, higher PTH levels may predict cortical deterioration [3]. In addition, serum calcium levels were not reduced; this lack of an effect can be attributed to secondary hyperparathyroidism in nephrectomy rats.

\section{Limitations}

First, this study utilized a hyperphosphatemia model that is not typically used since CKD patients would not be advised to increase phosphorus load. A low phosphorus diet has been found to suppress secondary hyperparathyroidism in mice with both mild and heavy proteinuria [52]. While we agree that it would be ideal to lower dietary phosphorus to prevent the development of hyperphosphatemia and the worsening of secondary hyperparathyroidism, patients, especially children with CKD, do not always follow low phosphorus intake recommendations due to food being unpalatable. Hence, the use of phosphate binding agents is frequently used. Our experimental design investigates the skeletal effects of hyperphosphatemia and severe secondary hyperparathyroidism in kidney disease. Dietary phosphorus restriction was not used in the $\mathrm{Nx}$ Control group to compare the mild increase in PTH to severe secondary hyperparathyroidism. Second, the highly-scattered plots in Fig. 7 could be due to several reasons including the small number of replicates per group in the study, the biological variation in disease status in different animals, and the dilution of the relative contribution of measured parameters in CKD due to the involvement of multiple signaling pathways. Third, the cause and effect relationship between changes in PTH and FGF23 levels and trabecular bone microstructure in response to nephrectomy and high phosphorous diet remain to be investigated.

\section{Conclusion}

Our study investigated the negative effects of renal failure and its relationship with phosphorus, FGF23, PTH, sclerostin, and bone. We found that high phosphorus diet adversely affects trabecular bone, whereas nephrectomy induced cortical bone loss. Future studies are needed to investigate the molecular mechanisms by which high phosphorus diet affects trabecular bone mass in models of CKD.

\section{Abbreviations \\ 1,25[OH] $]_{2}$ D3: 1,25-dihydroxy vitamin D3; BMD: Bone mineral density; BV: Bone volume; BV/TV: Bone volume adjusted for tissue volume; CKD: Chronic kidney disease; DXA: Dual-energy X-ray absorptiometry; FGF23: Fibroblast growth factor 23; iPTH: Intact parathyroid hormone; Oc.Pm: Osteoclast perimeter; Oc.S/BS: Osteoclast surface adjusted for bone surface; PTH: Parathyroid hormone; rHPT: Renal hyperparathyroidism; SMI: Structure model index; TRAP: Tartrate-resistant acid phosphatase; TV: Tissue volume; vBMD: Volumetric bone mineral density; Wnt: Wingless- type MMTV integration site family}

\section{Acknowledgements}

We thank Nancy Lowen for technical assistance. This research was supported by funds from the Department of Pediatrics, Loma Linda University. SM's salary was supported by a senior research career scientist award from the United States Department of Veterans Affairs. The research work was performed at facilities provided by the US Department of Veterans Affairs.

\section{Funding}

This research was supported by funds from the Department of Pediatrics, Loma Linda University. SM's salary was supported by a senior research career scientist award from the United States Department of Veterans Affairs.

\section{Availability of data and materials}

Data that support the findings of this study are available upon request from the corresponding author.

\section{Authors' contributions}

NMB: data analysis and writing the manuscript; CPS: study design, experimental procedures, data analysis, and writing the manuscript; RCL: data analysis; HW: experimental procedures; SM: study design, data analysis, and writing the manuscript. All authors read and approved the final manuscript.

\section{Ethics approval}

Animal housing and procedures were approved by the Institutional Animal Care and Use Committee of the Jerry L. Pettis Memorial Veterans Affairs Medical Center. Rats were anesthetized with isoflurane prior to surgical procedures. All procedures performed complied with the principles and procedures of the Guidelines for the Care and Use of Experimental Animals.

Consent for publication

Not applicable.

Competing interests

The authors declare that they have no competing interests.

\section{Publisher's Note}

Springer Nature remains neutral with regard to jurisdictional claims in published maps and institutional affiliations.

\section{Author details}

${ }^{1}$ Musculoskeletal Disease Center, VA Loma Linda Healthcare System, 11201 Benton Street, Loma Linda, CA 92357, USA. ²Department of Pediatrics, Loma Linda University, Loma Linda, CA 92354, USA. ${ }^{3}$ Department of Medicine, Loma Linda University, Loma Linda, CA 92354, USA. ${ }^{4}$ Department of Orthopedic

Surgery, Loma Linda University, Loma Linda, CA 92354, USA.

Received: 1 June 2017 Accepted: 21 January 2018

Published online: 02 February 2018

\section{References}

1. USRDS annual data report. Epidemiology of kidney disease in the United States. Bethesda, MD: National Institutes of Health, National Institute of Diabetes and Digestive and Kidney Diseases; 2015.

2. Lau WL, Linnes M, Chu EY, Foster BL, Bartley BA, Somerman MJ, et al. High phosphate feeding promotes mineral and bone abnormalities in mice with 
chronic kidney disease. Nephrol Dial Transplant. 2013;28(1):62-9. https:/doi. org/10.1093/ndt/gfs333.

3. Nickolas TL, Stein EM, Dworakowski E, Nishiyama KK, Komandah-Kosseh M, Zhang CA, et al. Rapid cortical bone loss in patients with chronic kidney disease. J Bone Miner Res. 2013;28(8):1811-20. https:/doi.org/10.1002/jbmr.1916.

4. Ott SM. Bone histomorphometry in renal osteodystrophy. Semin Nephrol. 2009;29(2):122-32. https://doi.org/10.1016/j.semnephrol.2009.01.005.

5. Spasovski GB, Bervoets AR, Behets GJ, Ivanovski N, Sikole A, Dams G, et al. Spectrum of renal bone disease in end-stage renal failure patients not yet on dialysis. Nephrol Dialysis, transplantation. 2003;18(6):1159-66.

6. Naylor KL, MCArthur E, Leslie WD, Fraser LA, Jamal SA, Cadarette SM, et al. The three-year incidence of fracture in chronic kidney disease. Kidney Int. 2014;86(4):810-8. https://doi.org/10.1038/ki.2013.547.

7. Miller MA, Chin J, Miller SC, Fox J. Disparate effects of mild, moderate, and severe secondary hyperparathyroidism on cancellous and cortical bone in rats with chronic renal insufficiency. Bone. 1998;23(3):257-66.

8. Shipov A, Segev G, Meltzer H, Milrad M, Brenner O, Atkins A, et al. The effect of naturally occurring chronic kidney disease on the micro-structural and mechanical properties of bone. PLoS One. 2014;9(10):e110057. https://doi. org/10.1371/journal.pone.0110057.

9. Heveran CM, Ortega AM, Cureton A, Clark R, Livingston EW, Bateman TA, et al. Moderate chronic kidney disease impairs bone quality in C57BI/6J mice. Bone. 2016:86:1-9. https://doi.org/10.1016/..bone.2016.02.006.

10. Iwasaki Y, Kazama JJ, Yamato H, Matsugaki A, Nakano T, Fukagawa M. Altered material properties are responsible for bone fragility in rats with chronic kidney injury. Bone. 2015;81:247-54. https://doi.org/10.1016/j.bone.2015.07.015.

11. Iwasaki Y, Kazama JJ, Yamato H, Fukagawa M. Changes in chemical composition of cortical bone associated with bone fragility in rat model with chronic kidney disease. Bone. 2011;48(6):1260-7. https://doi.org/10.1016/j.bone.2011.03.672.

12. Stein EM, Silva BC, Boutroy S, Zhou B, Wang J, Udesky J, et al. Primary hyperparathyroidism is associated with abnormal cortical and Trabecular microstructure and reduced bone stiffness in postmenopausal women. J Bone Miner Res. 2013;28(5):1029-40. https://doi.org/10.1002/jbmr.1841.

13. Silva BC, Boutroy S, Zhang C, McMahon DJ, Zhou B, Wang J, et al. Trabecular bone score (TBS)-a novel method to evaluate bone microarchitectural texture in patients with primary hyperparathyroidism. J Clin Endocrinol Metab. 2013: 98(5):1963-70. https://doi.org/10.1210/jc.2012-4255.

14. Wetzsteon RJ, Kalkwarf HJ, Shults J, Zemel BS, Foster BJ, Griffin L, et al. Volumetric bone mineral density and bone structure in childhood chronic kidney disease. J Bone Miner Res. 2011;26(9):2235-44. https:// doi.org/10.1002/jbmr.427.

15. Tsampalieros A, Kalkwarf HJ, Wetzsteon RJ, Shults J, Zemel BS, Foster BJ, et al. Changes in bone structure and the muscle-bone unit in children with chronic kidney disease. Kidney Int. 2013;83(3):495-502. https://doi.org/10. 1038/ki.2012.347.

16. Gentry JWJ, Davenport D, Malluche HH. Serum phosphorus adds to value of serum parathyroid hormone for assessment of bone turnover in renal osteodystrophy. Clin Nephrol. 2016;86(7):9-17.

17. Brown EM, Gamba G, Riccardi D, Lombardi M, Butters R, Kifor O, et al. Cloning and characterization of an extracellular Ca2+-sensing receptor from bovine parathyroid. Nature. 1993;366(6455):575-80.

18. Newman CL, Chen NX, Smith E, Smith M, Brown D, Moe SM, et al. Compromised vertebral structural and mechanical properties associated with progressive kidney disease and the effects of traditional pharmacological interventions. Bone. 2015;77:50-6. https://doi.org/10. 1016/j.bone.2015.04.021

19. Isakova T, Wahl P, Vargas G, Gutiérrez OM, Scialla J, Xie H, et al. FGF23, PTH and phosphorus metabolism in the chronic renal insufficiency cohort. Kidney Int. 2011;79(12):1370-8. https://doi.org/10.1038/ki.2011.47.

20. Lavi-Moshayoff V, Wasserman G, Meir T, Silver J, Naveh-Many T. PTH increases FGF23 gene expression and mediates the high-FGF23 levels of experimental kidney failure: a bone parathyroid feedback loop. Am J Physiol Renal Physiol. 2010;299(4):F882-9. https://doi.org/10. 1152/ajprenal.00360.2010.

21. Martin A, David V, Quarles LD. Regulation and function of the FGF23/ klotho endocrine pathways. Physiol Rev. 2012;92(1):131-55. https://doi. org/10.1152/physrev.00002.2011.

22. Sanchez CP, Salusky IB, Kuizon BD, Abdella P, Juppner H, Goodman WG Growth of long bones in renal failure: roles of hyperparathyroidism, growth hormone and calcitriol. Kidney Int. 1998;54(6):1879-87. https://doi.org/10. 1046/j.1523-1755.1998.00199.x.
23. Yu H, Watt H, Kesavan C, Johnson PJ, Wergedal JE, Mohan S. Lasting consequences of traumatic events on behavioral and skeletal parameters in a mouse model for post-traumatic stress disorder (PTSD). PLoS One. 2012;7(8):e42684. https://doi.org/10.1371/journal.pone.0042684.

24. Masinde GL, Li X, Gu W, Wergedal J, Mohan S, Baylink DJ. Quantitative trait loci for bone density in mice: the genes determining total skeletal density and femur density show little overlap in F2 mice. Calcif Tissue Int. 2002; 71(5):421-8. https://doi.org/10.1007/s00223-001-1113-z.

25. Yu H, Watt $\mathrm{H}$, Kesavan $\mathrm{C}$, Mohan $\mathrm{S}$. The negative impact of single prolonged stress (SPS) on bone development in mice. Stress (Amsterdam, Netherlands). 2013;16(5):564-70. https://doi.org/10.3109/10253890.2013.806908.

26. Kesavan C, Mohan S. Bone mass gained in response to external loading is preserved for several weeks following cessation of loading in 10 week C57BL/6J mice. J Musculoskelet Neuronal Interact. 2010;10(4):274-80.

27. Cheng S, Xing W, Pourteymoor S, Schulte J, Mohan S. Conditional deletion of Prolyl Hydroxylase domain-containing protein 2 (Phd2) gene reveals its essential role in Chondrocyte function and Endochondral bone formation. Endocrinology. 2016;157(1):127-40. https://doi.org/10.1210/en.2015-1473.

28. Dempster DW, Compston JE, Drezner MK, Glorieux FH, Kanis JA, Malluche H, et al. Standardized nomenclature, symbols, and units for bone Histomorphometry: a 2012 update of the report of the ASBMR Histomorphometry nomenclature committee. J Bone Miner Res. 2013;28(1):2-17. https//doi.org/10.1002/jbmr.1805.

29. Hopper TA, Wehrli FW, Saha PK, Andre JB, Wright AC, Sanchez CP, et al. Quantitative microcomputed tomography assessment of intratrabecular, intertrabecular, and cortical bone architecture in a rat model of severe renal osteodystrophy. J Comput Assist Tomogr. 2007;31 (2):320-8.

30. Negri A, Lombas C, Cuevas C, Schiavelli R, Bogado C, Zanchetta J, Evaluation of cortical bone by peripheral quantitative computed tomography in renal transplant recipients. Transplant Proc. 2005;37(2):1020-22. Elsevier.

31. Neves KR, Graciolli FG, dos Reis LM, Pasqualucci CA, Moyses RM, Jorgetti V. Adverse effects of hyperphosphatemia on myocardial hypertrophy, renal function, and bone in rats with renal failure. Kidney Int. 2004;66(6):2237-44. https://doi.org/10.1111/j.1523-1755.2004.66013.x.

32. Aubin JE, Bonnelye E. Osteoprotegerin and its ligand: a new paradigm for regulation of osteoclastogenesis and bone resorption. Medscape Womens Health. 2000:5(2):5.

33. Takenaka T, Inoue T, Miyazaki T, Hayashi M, Suzuki H. Xeno-Klotho inhibits parathyroid hormone signaling. J Bone Miner Res. 2016:31(2):455-62. https://doi.org/10.1002/jbmr.2691.

34. Hercz G. Regulation of bone remodeling: impact of novel therapies. Semin Dial. 2001;14(1):55-60.

35. Mazzaferro S, Tartaglione L, Rotondi S, Pasquali M. Interaction between vitamin $\mathrm{D}$ and Calcimimetics in chronic kidney disease. In: Ureña Torres PA, Cozzolino $M$, Vervloet MG, editors. Vitamin D in chronic kidney disease. Cham: Springer International Publishing; 2016. p. 537-62

36. Stubbs JR, He N, Idiculla A, Gillihan R, Liu S, David V, et al. Longitudinal evaluation of FGF23 changes and mineral metabolism abnormalities in a mouse model of chronic kidney disease. J Bone Miner Res. 2012;27(1):38-46. https//doi.org/10.1002/ jbmr.516.

37. Prie $D$, Friedlander $G$. Reciprocal control of 1,25-dihydroxyvitamin $D$ and FGF23 formation involving the FGF23/Klotho system. Clin J Am Soc Nephrol. 2010;5(9):1717-22. https://doi.org/10.2215/cjn.02680310.

38. Olauson $H$, Lindberg K, Amin R, Jia T, Wernerson A, Andersson G, et al. Targeted deletion of Klotho in kidney distal tubule disrupts mineral metabolism. J Am Soc Nephrol. 2012:23(10):1641-51. https://doi.org/10.1681/asn.2012010048.

39. Sakan H, Nakatani K, Asai O, Imura A, Tanaka T, Yoshimoto S, et al. Reduced renal alpha-Klotho expression in CKD patients and its effect on renal phosphate handling and vitamin D metabolism. PLoS One. 2014;9(1): e86301. https://doi.org/10.1371/journal.pone.0086301.

40. Kurosu H, Ogawa Y, Miyoshi M, Yamamoto M, Nandi A, Rosenblatt KP, et al. Regulation of fibroblast growth factor-23 signaling by klotho. J Biol Chem. 2006;281(10):6120-3. https://doi.org/10.1074/jbc.C500457200.

41. Saji F, Shiizaki K, Shimada S, Okada T, Kunimoto K, Sakaguchi T, et al. Regulation of fibroblast growth factor 23 production in bone in uremic rats. Nephron Physiol. 2009;111(4):59-66. https://doi.org/10.1159/000210389.

42. Manghat P, Fraser WD, Wierzbicki AS, Fogelman I, Goldsmith DJ, Hampson G. Fibroblast growth factor-23 is associated with C-reactive protein, serum phosphate and bone mineral density in chronic kidney disease. Osteoporos Int. 2010;21(11):1853-61. https://doi.org/10.1007/s00198-009-1142-4.

43. Drake MT, Khosla S. Hormonal and systemic regulation of sclerostin. Bone. 2016;96:8-17. https://doi.org/10.1016/j.bone.2016.12.004. 
44. Bellido T, Ali AA, Gubrij I, Plotkin LI, Fu Q, O'Brien CA, et al. Chronic elevation of parathyroid hormone in mice reduces expression of sclerostin by osteocytes: a novel mechanism for hormonal control of osteoblastogenesis. Endocrinology. 2005;146(11):4577-83. https://doi.org/10. 1210/en.2005-0239.

45. Delgado-Calle J, Sato AY, Bellido T. Role and mechanism of action of sclerostin in bone. Bone. 2016;96:29-37. https://doi.org/10.1016/j.bone.2016. 10.007.

46. Gori F, Lerner U, Ohlsson C, Baron R. A new WNT on the bone: WNT16, cortical bone thickness, porosity and fractures. BoneKEy Reports. 2015;4:669. https://doi.org/10.1038/bonekey.2015.36

47. Carrillo-López N, Panizo S, Alonso-Montes C, Román-García P, Rodríguez I, Martínez-Salgado C, et al. Direct inhibition of osteoblastic Wnt pathway by fibroblast growth factor 23 contributes to bone loss in chronic kidney disease. Kidney Int. 2016;90(1):77-89. https://doi.org/10.1016/j.kint.2016.01.024.

48. Saracyn M, Czarzasta K, Brytan M, Murawski P, Lewicki S, Ząbkowski T, et al. Role of nitric oxide pathway in development and progression of chronic kidney disease in rats sensitive and resistant to its occurrence in an experimental model of 5/6 Nephrectomy. Med Sci Monit. 2017;23:4865-73. https://doi.org/10.12659/MSM.903820.

49. Campese VM, Kogosov E, Koss M. Renal afferent denervation prevents the progression of renal disease in the renal ablation model of chronic renal failure in the rat. Am J Kidney Dis. 1995;26(5):861-5. doi:https://doi.org/10. 1016/0272-6386(95)90456-5

50. Kasiske BL, Donnell MP, Garvis WJ, Keane WF. Pharmacologic treatment of hyperlipidemia reduces glomerular injury in rat 5/6 nephrectomy model of chronic renal failure. Circ Res. 1988;62(2):367.

51. Fratzl P, Gupta HS, Paschalis EP, Roschger P. Structure and mechanical quality of the collagen-mineral nano-composite in bone. J Mater Chem. 2004;14(14):2115-23. https://doi.org/10.1039/B402005G.

52. Bohnert BN, Daniel C, Amann K, Voelkl J, Alesutan I, Lang F, et al. Impact of phosphorus restriction and vitamin D-substitution on secondary hyperparathyroidism in a Proteinuric mouse model. Kidney Blood Press Res. 2015;40(2):153-65.

\section{Submit your next manuscript to BioMed Central and we will help you at every step:}

- We accept pre-submission inquiries

- Our selector tool helps you to find the most relevant journal

- We provide round the clock customer support

- Convenient online submission

- Thorough peer review

- Inclusion in PubMed and all major indexing services

- Maximum visibility for your research

Submit your manuscript at www.biomedcentral.com/submit 